\title{
Atenção Primária a Saúde voltada as necessidades dos idosos: da teoria à prática
}

\author{
Primary healthcare geared to the needs of the elderly: \\ from theory to practice
}

Aline Blaya Martins ${ }^{1}$ Otávio Pereira D'Avila ${ }^{2}$ Juliana Balbinot Hilgert ${ }^{3}$ Fernando Neves Hugo ${ }^{3}$
${ }^{1}$ Centro de Pesquisa em Odontologia Social, Universidade Federal do Rio Grande do Sul. R. Ramiro Barcelos 2492, Rio Branco. 90.035-003 Porto Alegre RS Brasil. fernandoneveshugo@ gmail.com

${ }^{2}$ Programa de Pós-

Graduação em Odontologia, Universidade Federal do Rio Grande do Sul.

${ }^{3}$ Departamento de

Odontologia Preventiva e

Social, Universidade Federal do Rio Grande do Sul.

\begin{abstract}
This article seeks to establish a confrontation between theory and practice with respect to healthcare for the elderly. The theory was evaluated by a study of documents that comprise the legal and theoretical framework related to the health of the elderly, which generated a matrix to assess universal access, equity, comprehensiveness and quality of care. However, the practice of healthcare was evaluated by a population-based study conducted on 862 elderly individuals and a census that assessed primary healthcare (PHC) units in two districts of Porto Alegre in the state of Rio Grande do Sul, Brazil. The variables chosen to verify the categories were type of care accessed, first contact attribute, wheelchair accessibility and availability of priority access. Other variables include comprehensive care and the presence of dental root remains, basic $\mathrm{PHC}$ score, the adequacy of heal th facilities and ongoing professional training, as well as cross-sectional categories, elderly groups and home care. A mismatch was found between recommendation and practice in some factors and advances were observed in the equity and quality of care regarding the ongoing training of professionals.

Key words Aging, Health services for the elderly, Public health policies, Primary healthcare, Access to healthcare
\end{abstract}

Resumo Oobjetivo deste artigo é estabelecer uma confrontação entre teoria e prática no que tange ao cuidado com a saúde de pessoas idosas. A teoria foi avaliada através de um estudo de documentos que compõem o arcabouço teórico-legal relacionado com a saúde do idoso e que gerou a construção de uma matriz com as seguintes categorias: Acesso Universal, Equidade, Integralidade e Qualidade do Cuidado. Já a prática foi avaliada por um estudo de base populacional realizado junto a 862 idosos $e$ por um censo que avaliou unidades de atenção primária (APS), ambos em dois distritos sanitários de Porto Alegre (RS). As variáveis que avaliaram as categorias foram respectivamente: tipo de serviço acessado, atributo de primeiro contato, acessibilidade de cadeirantes; presença de acesso prioritário; atributo integralidade e presença de restos radiculares; escore essencial da APS, adequação das estruturas das unidades de APS e capacitação profissional e variáveis transversais: grupos de idosos e visita domiciliar. Verificou-se um descompasso entre o preconizado e o estabelecido em alguns aspectos e observaram-se avanços em relação à equidade e à qualidade do cuidado em relação à capacitação dos profissionais.

Palavras-chave Envelhecimento, Serviços de saúde para idosos, Políticas públicas de saúde, Atenção Primária à Saúde, Acesso aos serviços de saúde 


\section{Introdução}

Mais de dez anos se passaram desde que a Política de Saúde do Idoso ${ }^{1}$ foi publicada e que a $2^{a}$ Conferência Mundial das Nações Unidas sobre o Envelhecimento aconteceu. Ambos os fatos foram relevantes em relação às políticas voltadas para a saúde do idoso. Em 2002, em Madri, Espanha, foi lançado o Plano Internacional de ações sobre o Envelhecimento, que apontou mudanças que deveriam ser feitas para que os idosos pudessem ter o direito de participar de uma sociedade mais inclusiva e digna ${ }^{2}$. Na década que passou, foi possível observar uma mudança na expectativa de vida e também no movimento em torno de políticas no afã de proporcionar o envelhecimento ativo da população $0^{2-9}$.

No Brasil, percebe-se um crescimento acelerado dos idosos na pirâmide etária. A participação relativa desta população, que era de $4,8 \%$ em 1991, chegou a $7,4 \% \mathrm{em} 2010^{10}$. Sendo que as regiões Sudeste e Sul apresentam evolução semelhante na estrutura etária, mantendo-se como as mais envelhecidas do país. Tais cidadãos possuem o direito de utilizar o Sistema Único de Saúde (SUS) de forma equitativa e integral. Os princípios do SUS foram conquistados no momento da promulgação da Constituição Federal e vêm sendo reforçados como direito inalienável e como diretriz fundamental na gestão do cuidado à saúde do idoso ${ }^{3,5-7,11}$.

A fim de qualificar o cuidado, o SUS está orientado para a Atenção Primária à Saúde (APS) ${ }^{12}$. O Pacto pela Vida e a Política Nacional de Saúde da Pessoa Idosa (PNSPI), ambos de 2006, definiram que a atenção à saúde dessa população deve ter como porta de entrada a APS/Saúde da Família (SF), tendo como referência a rede de serviços especializada de média e alta complexidade ${ }^{6}$. Além disso, a saúde da população idosa também passou a ser uma prioridade no SUS e, por conseguinte, da Estratégia de Saúde da Família (ESF), modelo fortemente embasado nos atributos da APS e que busca a qualificação dessa atenção $0^{5,12}$.

Parte-se, portanto, de um panorama otimista: um país com um enorme contingente de idosos cobertos por um sistema de saúde com princípios sólidos e por políticas de atenção à sua saúde que buscam atender às suas necessidades conforme a realidade local e seguindo políticas globais ${ }^{5,7,9}$. No entanto, segundo a própria $\mathrm{PNSPI}^{6}$, ainda há muito a ser feito para que o SUS dê respostas efetivas e eficazes às necessidades e demandas de saúde da população idosa brasileira. Evidências demonstram problemas relacionados com o uso e o acesso aos serviços de saúde e inadequação do modelo de atenção para atender a demanda dos idosos ${ }^{13}$ : alta prevalência de sintomatologia de depressão, cognição diminuída e graus de dependência diversos, além de múltiplos diagnósticos e uso de polifarmácia ${ }^{14}$.

É possível que a confrontação entre categorias extraídas do arcabouço teórico-legal que aborda a saúde do idoso e a verificação da extensão do cuidado em APS provido para essa faixa etária de dois distritos sanitários (DS) de Porto Alegre, possa apontar aspectos de convergência e de distanciamento entre teoria e prática. O objetivo deste estudo foi estabelecer uma confrontação entre teoria e prática no que tange ao cuidado voltado às necessidades de saúde de pessoas idosas em dois DS de Porto Alegre.

\section{Metodologia}

Este estudo foi aprovado pelo Comitê de Pesquisa e Ética da Faculdade de Odontologia da UFRGS.

Diante do objetivo proposto, a metodologia será descrita em 4 etapas: 1a- Estudo teórico, que visa avaliar o que a teoria relacionada com a saúde do idoso preconiza; 2a ${ }^{\mathrm{a}}$ Estudo epidemiológico de base populacional; 3a- Censo de Avaliação das Unidades de Saúde. Sendo estas duas etapas responsáveis por fornecer dados empíricos capazes de avaliar o que ocorre na prática; e, $4^{\text {a }}$ - Confrontação.

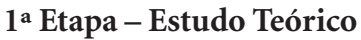

\section{Levantamento de documentos}

A busca de documentos parte da seguinte questão: Considerando os princípios do SUS, quais são as categorias comuns abordadas em políticas e protocolos nacionais e internacionais relacionados com a saúde do idoso?

A estratégia de busca de documentos foi feita através dos seguintes unitermos: "Política", "atenção primária à saúde" e "idosos", e seus correspondentes em inglês e espanhol. Além destes, foram incluídos: "Ministério da Saúde", "World Health Organization-WHO", "Panamerican Health Organization- PAHO". Os limites temporais da busca foram os anos de 1999 a 2012. A autoria dos documentos deveria ser de instituições/órgãos responsáveis por publicações de políticas públicas e protocolos.

Observados os critérios de busca, foram selecionados 5 documentos nacionais e 4 internacionais. Dentre os nacionais, 4 eram politicas relacionadas com os idosos e um era um protocolo 
para APS ${ }^{3,5-7,9}$. Também foram selecionados para a análise 3 documentos internacionais, 2 políticas e um protocolo de cuidados com a saúde do idoso propostos pela Organização Mundial de Saúde $(\mathrm{OMS})^{2,4,8}$.

\section{Escolha das categorias}

Sobre a análise do conteúdo dos documentos, dois tipos de unidade foram utilizados: a de registro e a de contexto. Na primeira, foram selecionados temas específicos do conteúdo através de palavras ou expressões que mostraram ser afins. Em outros casos, foi necessário analisar o contexto em que a temática estava colocada a fim de observar se os conceitos relacionados com as categorias não estavam postos de uma forma descritiva.

Contudo, a etapa crucial para a construção de categorias, aconteceu após a análise das informações. Num primeiro momento, as categorias brotaram da detecção de aspectos que surgiam com regularidade e que são princípios básicos do arcabouço teórico-legal do SUS ${ }^{11,15}$. Contudo, às categorias iniciais foi acrescida mais uma com o desenrolar do estudo em decorrência da regularidade com que pôde ser observada e devido à sua relevância.

Desta forma, foram escolhidas as seguintes categorias: acesso universal, equidade, integralidade e qualidade no cuidado à saúde do idoso.

\section{Construção da Matriz Teórico-Lógica}

Após a escolha das categorias e nova análise dos documentos para sistematização da forma como as categorias estavam apresentadas, uma matriz teórico-lógica de análise foi desenvolvida como forma de registro.

\section{2a Etapa - Estudo Epidemiológico de Base Populacional}

\section{Coleta de Dados}

Um estudo de base populacional, de delineamento transversal, foi realizado a fim de verificar a extensão em que o cuidado é produzido conforme condições referidas pelos idosos moradores dos DS Lomba do Pinheiro e Partenon de Porto Alegre, Brasil.

Segundo o Censo IBGE, Porto Alegre em 2010 tinha 1.409.939 habitantes, sendo 211.986 pessoas com 60 anos ou mais. Nos referidos DS, a população estimada com 60 anos ou mais é de 12.871 indivíduos ${ }^{10}$.

Idosos que vivem na comunidade de forma independente, com 60 anos ou mais, moradores dos DS Lomba e Partenon, em Porto Alegre, foram convidados a participar do estudo por meio de uma amostra por conglomerados.

Critério de inclusão: ter idade $\geq 60$ anos, domicílio localizado nos referidos DS, ser independente e sem déficit cognitivo segundo o teste de rastreamento Mini Exame do Estado Mental $(\mathrm{MEEM})^{16,17}$. A fim de rastrear possíveis danos cognitivos que comprometessem a qualidade dos dados, foram utilizados diferentes pontos de corte como critério de inclusão. Ao realizar-se a soma das respostas dos 30 itens que compõem o MEEM, o número mínimo de acertos para inclusão foi de: 13 - analfabetos, 18 - escolaridade média e 26-alta escolaridade ${ }^{17}$. Desta forma, somente aqueles que preencheram os critérios e assinaram o Termo de Consentimento Livre e Esclarecido (TCLE), foram convidados a responder os demais instrumentos.

Este estudo faz parte de uma pesquisa que teve como desfecho a autopercepção de saúde dos idosos. O cálculo amostral, portanto, usou os parâmetros de prevalência de boa percepção de saúde bucal.

Foi realizado um estudo piloto com 50 idosos moradores dos mesmos DS para estimação do tamanho amostral. Os setores censitários utilizados para o estudo piloto foram excluídos do sorteio posterior. O cálculo amostral foi realizado considerando a prevalência de autopercepção de saúde bucal boa que foi igual a 59\%. Estimou-se que a razão de prevalência dos idosos que dispunham de uma APS adequada era de 1,32, conforme o escore essencial do Instrumento de Avaliação da Atenção Primária (PCATool Brasil) ${ }^{18}$. Assim, selecionado um $\alpha$ bidirecional de 0,05 e um $\beta$ de 0,20 , foi estimada uma amostra total de 454 idosos. Foram acrescentados $10 \%$ para possíveis perdas ou recusas e $15 \%$ para controle de fatores de confusão. $\mathrm{Na}$ falta de uma estimativa precisa para minimizar o efeito do delineamento, a amostra foi ampliada 1,5 vez de modo a realizar as análises ajustadas. Chegou-se, assim, ao número de 883 indivíduos.

A amostragem foi realizada através da metodologia proposta por Barros e Victora ${ }^{19}$ para avaliação de conglomerados (setores censitários). A quantidade de entrevistas por setor foi estimado a partir do número de moradores em cada domicílio e da proporção de idosos/setor censitário de Porto Alegre em 2000. Tais informações foram cedidas pela Coordenação Geral de Vigilância em Saúde do Município de Porto Alegre (CGVS). A partir destes dados obteve-se um número de 30 entrevistas por setor censitário e, portanto, foram excluídos aqueles com menos de 30 idosos (75 setores). Dentro de cada DS, os setores foram amostrados utilizando uma amostragem sistemática. Inicialmente, foi 
realizado um sorteio de 35 setores dentre os 240 que compõem a área de abrangência dos dois DS. O número de setores censitários sorteados foi proporcional ao número total que compõem cada um dos distritos estudados e buscou-se, também, distribuir os setores sorteados proporcionalmente de acordo com o número que compunha o território adscrito de cada unidade de APS.

A definição do quarteirão elencado como ponto de partida para a coleta de dados foi baseada em um fluxograma elaborado a partir dos mapas dos setores. Os quarteirões foram numerados e o primeiro foi sorteado seguindo-se uma sequência a partir deste. Em cada quarteirão também foi sorteada aleatoriamente uma esquina que representou o ponto inicial.

A partir da esquina inicial, as visitas seguiram a seguinte ordem: partindo da primeira casa, os pesquisadores seguiram sempre para a esquerda de frente para as casas. Após a realização da entrevista, um domicílio foi pulado e a próxima casa foi visitada, até que 30 entrevistas referentes aquele setor censitário tivessem sido realizadas ou que os domicílios do setor tivessem sido todos visitados de acordo com os padrões estabelecidos.

A equipe de coleta foi composta por 10 estudantes de odontologia com mínimo de 6 semestres completados de graduação e 3 cirurgiões-dentistas alunos de pós-graduação. Um treinamento de $40 \mathrm{hs}$ e um estudo piloto foram realizados previamente à coleta.

O treinamento foi organizado da seguinte maneira: i) estudo das bases teóricas que compõem o marco referencial; ii) estudo dos instrumentos; iii) discussão sobre o processo de amostragem; iv) apropriação da tecnologia Personal Digital Assistant (PDA) e do software desenvolvido; v) aulas sobre condições de saúde bucal dos idosos para a execução do exame bucal; vi) discussão de casos complexos; vi) simulação de entrevistas e exames. Posteriormente, o estudo piloto foi realizado e a calibração foi feita. O acompanhamento do processo de coleta de dados se deu semanalmente para assegurar a qualidade dos dados.

A coleta de dados foi executada nos domicílios durante os meses de fevereiro de 2010 a fevereiro de 2011.

\section{Medidas}

Para o questionário individual foram coletados dados a respeito de sexo, idade, renda, número de dependentes, percepção de que a renda era suficiente para a família e escolaridade. Para fins de análise, a renda foi categorizada em: " $\leq 2$ salários mínimos" e "> 2 salários mínimos". A percepção de que a renda é suficiente para as despesas familiares foi coletada com respostas dicotômicas.

Em relação às condições de saúde, foi empregado o Mini Exame do Estado Mental (MEEM). Trata-se um instrumento de rastreamento de possíveis déficits cognitivos, desenvolvido por Folstein et al. ${ }^{16} \mathrm{e}$ validado para o português (BR) por Bertolucci et al. ${ }^{17}$.

Dentre os indivíduos incluídos na amostra, para a análise da função cognitiva, maiores escores foram considerados como melhor desempenho cognitivo. Para tanto as médias dos escores do MEEM de cada nível de escolaridade foram obtidas e uma variável foi criada estabelecendo a média, de acordo com escolaridade baixa, media ou alta, como ponto de corte. Foram então definidas as seguintes categorias: acima da média do MEEM - Capacidade cognitiva alta, e abaixo da média do MEEM - Capacidade cognitiva baixa.

A Escala de Depressão Geriátrica-GDS, criada por Yesavage et al. ${ }^{20} \mathrm{e}$ validada para o português (BR) por Almeida e Almeida ${ }^{21}$, costuma ser utilizada na avaliação da sintomatologia depressiva em idosos. Sua versão de 15 itens (GDS-15) foi utilizada nesta avaliação. Trata-se de uma escala de fácil utilização, com respostas de "sim" ou "não", que devem ser somadas para compor um escore. O escore é então dicotomizado, sendo: $\leq 5$ pontos, sem sintomatologia depressiva; $\mathrm{e} \geq 6$ pontos, com sintomatologia depressiva.

As condições referidas de saúde diabetes, hipertensão e dor nas costas foram coletadas a partir da condição referida através da seguinte pergunta: "Das doenças que vou lhe perguntar a seguir, responda sim caso algum médico já tenha lhe dito que o(a) senhor(a) tem ou tenha tido".

O exame odontológico foi realizado com luz natural, de acordo com a metodologia proposta pela $\mathrm{OMS}^{22}$. Neste exame foi avaliado o número de dentes e de restos radiculares. O número de dentes foi categorizado como ${ }^{23}: 0,1-19, \geq 20$ dentes. Os restos radiculares foram dicotomizados como: " 0 " ou "pelo menos 1".

O PCATooL Brasil - Usuário foi o Instrumento de Avaliação da APS $^{24,25}$ validado para o idioma português (BR) e proposto pelo Ministério de Saúde ${ }^{18}$ como instrumento de avaliação da qualidade de serviços de saúde de APS.

O instrumento é composto por 55 itens divididos em 10 componentes (subescalas). As respostas são do tipo Likert variando de 1 (com certeza não) a 4 (com certeza sim). O instrumento atribui, através das subescalas, escores para cada atributo essencial, a saber: acesso de primeiro contato, longitudinalidade, integralidade e coordenação do 
cuidado. E para os atributos derivados: orientação familiar e orientação comunitária.

Os escores são capazes de avaliar o grau de orientação do serviço para a APS em relação aos atributos. Além disso, um escore essencial pode ser gerado através da análise de todas as questões que compõem os escores dos atributos essenciais. $\mathrm{E}$ um escore geral é estabelecido a partir dos escores dos atributos essenciais e derivados. Escores altos denotam efetividade da APS. O escore de cada atributo foi categorizado de acordo com o ponto de corte preconizado ${ }^{18}$ : i) Serviços com orientação para a APS- com escores $\geq 6,6$ (num intervalo de 0 a 10). ii) Serviços sem orientação para APS- com escores $<6,6$.

\section{Uso dos serviços}

A população de idosos que foi amostrada neste estudo mora em domicílios adscritos aos DS Lomba do Pinheiro e Partenon, que possuem 19 unidades de atenção primária responsáveis pela atenção à saúde dos idosos e de seus familiares. Além disso, um hospital privado que presta serviços ao SUS e diversas clínicas e consultórios de convênios e particulares também ofertam seus serviços a esta população.

Para avaliar o tipo de serviço utilizado em relação à saúde geral e à saúde bucal e satisfação com os mesmos, foram utilizados dados provenientes de um questionário com informações sobre os serviços acessados.

A variável "tipo de serviços de saúde acessado" foi utilizada conforme o padrão proposto para avaliar a afiliação do usuário no instrumento PCATool Brasil ${ }^{18}$. Desta forma, a afiliação foi estabelecida segundo três questões que se referiam a: qual serviço de saúde o idoso costumava acessar, qual serviço o idoso acreditava que lhe conheciam melhor como pessoa e qual serviço o idoso acreditava ser o mais responsável pelo seu atendimento. De acordo com o maior número de respostas iguais, e seguindo outros critérios propostos para os casos em que respostas eram diferentes, estabeleceu-se a qual serviço o idoso estava vinculado. Posteriormente, este serviço foi classificado de acordo com o seu tipo, conforme uma tabela de referência. Desta forma, os serviços foram classificados como: UBS; USF; Pronto atendimento; Emergência de um hospital; Ambulatório de um hospital; Consultório de determinado médico; Ambulatório de um plano de saúde; Serviço de saúde no trabalho ou escola; e, Outro tipo de serviço.

Para fins de análise estatística os tipos de serviços foram categorizados como: UBS, USF e outros.
$\mathrm{Na}$ análise de serviços de saúde bucal, também foi incluída a categoria "nunca foi ao dentista".

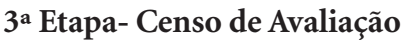 das Unidades de Saúde}

Todas as unidades de saúde (US) dos DS em funcionamento no primeiro semestre de 2011 tiveram seus ambientes físicos e dispositivos de acesso checados. Tais unidades compunham à época um universo de 19 unidades de saúde: 11 USF e 8 UBS.

\section{Medidas}

Foram utilizados os instrumentos desenvolvidos pela $\mathrm{OMS}^{7}$, baseados nos princípios de design universal: i) Ficha cadastral da US - possuía o nome da US, nome do coordenador, tempo decorrido desde o início do funcionamento da US, ou da última reforma realizada, e a data da auditoria; ii) Lista de checagem para a auditoria da acessibilidade das estruturas físicas em Centros de APS: instrumento composto por 44 itens, a maioria com verificação simples (sim/não, ou valor absoluto de largura/altura); iii) Lista de checagem para a auditoria da sinalização dos Centros de APS: instrumento composto por 20 itens.

Foram consideradas “inadequadas", em relação ao acesso e/ou sinalização, as US onde menos de $50 \%$ dos itens estavam de acordo com o preconizado, "adequadas", as US onde todos os parâmetros estavam conforme o preconizado, e "parcialmente adequadas" aquelas US que apresentavam entre 50 e $100 \%$ de itens adequados.

As seguintes questões foram integradas à avaliação das US: "Algum membro da equipe já realizou alguma capacitação/especialização sobre cuidados para com a saúde do idoso? Quantos?" As respostas foram categorizadas em: $0,1 \mathrm{ou} \geq 2$; "Existe algum tipo de acesso facilitado para idosos? Qual?" As respostas foram categorizadas em: realiza ou não agendamento por telefone; As próximas perguntas possuíam respostas dicotômicas (sim/ não): "A US disponibiliza algum grupo de idosos para os seus usuários?"; "A equipe de saúde realiza visitas domiciliares a acamados?”; "Há consultório odontológico na US em funcionamento? É acessível para cadeirantes?".

\section{Análise dos dados do Estudo de Base Populacional e do Censo das Unidades de Saúde}

Os dados foram tabulados e analisados no software de estatística SPSS 18.0 (SPSS Inc., USA). As variáveis quantitativas foram descritas na forma de média e respectivos desvios-padrão, enquanto 
as variáveis categóricas foram descritas na forma de frequência absoluta e relativa.

As características da amostra do estudo de base populacional foram apresentadas de duas formas: i) incluindo todos os participantes da amostra e ii) incluindo apenas os idosos que utilizavam US de APS.

A confiabilidade das escalas MEEM, GDS-15 e subescalas que compõem o PCATool Brasil foi testada por meio do Coeficiente $\alpha$ de Cronbach.

\section{$4^{\text {a }}$ Etapa - Confrontação}

De acordo com as categorias escolhidas para compor a matriz teórico-lógica, uma série de variáveis empíricas, que se enquadravam dentro do que compete a cada categoria, foi selecionada a fim de aferir a extensão que o cuidado é realizado na prática, quando comparamos ao que é preconizado. Tais variáveis podem ser observadas no Quadro 1.

\section{Resultados}

Os resultados deste estudo também serão apresentados em 4 etapas: $1^{\text {a }}$ - Resultados da Matriz teórico-lógica, 2a - Estudo de base populacional, $3^{\mathrm{a}}$ - Censo das US, e $4^{\mathrm{a}}$ - Confrontação.

\section{1 ${ }^{\text {a }}$ Etapa - \\ Resultados da Matriz Teórico-lógica}

A matriz teórico-lógica foi construída a partir da descrição da forma como cada um dos documentos abordou as categorias em análise (Quadros 2 e 3).

Durante tal construção foi possível observar que houve três distintas abordagens: i) a categoria foi abordada como um marco teórico no documento, ii) a categoria era o foco ou uma diretriz, vista com extrema relevância na construção do documento, e iii) ausência de qualquer referência à categoria.

Através da matriz, que está disposta nos Quadros 2 e 3, é possível observar que os princípios de universalidade, equidade e integralidade são marcos, ou diretrizes, abordados em todos os documentos, com exceção dos internacionais que possuem o foco na organização da APS. A qualidade do cuidado também foi uma categoria de análise relevante nos documentos avaliados.

\section{2a Etapa- \\ Resultados do Estudo de base populacional}

Foram convidados a participar deste estudo 994 idosos residentes nos DS. Destes, 83 não foram encontrados nos domicílios no dia do exame e não conseguiram ser agendados após 3 tentativas, 26 se recusaram a participar e 23 não atingiram os pontos de corte do MEEM. A amostra do estudo, portanto, foi composta por 862 pessoas que estavam aptas a compô-la, aceitaram participar e assinaram o TCLE. Em relação à saúde bucal, 52 idosos (cerca de 5\% da amostra) não aceitaram participar do exame odontológico e foram excluídos desta análise.

A amostra estudada foi composta por 260 (32\%) homens, com média de idade de 70 anos $( \pm$

Quadro 1. Descrição da correlação estabelecida entre as categorias e as variáveis empíricas aferidas.

\begin{tabular}{|c|c|c|c|c|}
\hline Categorias & Acesso universal & Equidade & Integralidade & Qualidade do cuidado \\
\hline \multirow{5}{*}{$\begin{array}{l}\text { Variáveis } \\
\text { empíricas } \\
\text { aferidas }\end{array}$} & $\begin{array}{l}\text { Tipo de serviço } \\
\text { acessado }\end{array}$ & $\begin{array}{c}\text { Presença de } \\
\text { acesso prioritário }\end{array}$ & $\begin{array}{c}\text { Atributo integralidade } \\
\text { - PCATool }\end{array}$ & $\begin{array}{l}\text { Escore essencial } \\
\text { - PCATool }\end{array}$ \\
\hline & $\begin{array}{l}\text { Atributo de primeiro } \\
\text { contato } \\
\text {-PCATool }\end{array}$ & & $\begin{array}{l}\text { Necessidades de } \\
\text { tratamento em } \\
\text { Saúde Bucal - restos } \\
\text { radiculares }\end{array}$ & $\begin{array}{l}\text { Adequação das estruturas } \\
\text { das Unidades de Saúde }\end{array}$ \\
\hline & $\begin{array}{l}\text { Acessibilidade } \\
\text { de cadeirantes } \\
\text { a consultórios } \\
\text { odontológicos }\end{array}$ & & & $\begin{array}{l}\text { Capacitação profissional: } \\
\text { Atenção à saúde do idoso }\end{array}$ \\
\hline & \multicolumn{4}{|c|}{ Grupo de idosos } \\
\hline & \multicolumn{4}{|c|}{ Visita domiciliar } \\
\hline
\end{tabular}


Quadro 2. Análise documental das políticas e protocolos nacionais e internacionais que envolvem a saúde do idoso, publicadas entre 2002-2006.

\begin{tabular}{|c|c|c|c|c|}
\hline & Acesso universal & Equidade & Integralidade & Qualidade do cuidado \\
\hline $\begin{array}{l}\text { Envelhecimento } \\
\text { Ativo (WHO, } \\
2002)^{2}\end{array}$ & $\begin{array}{l}\text { Livre acesso a postos } \\
\text { de saúde e demais } \\
\text { níveis de atenção - } \\
\text { apresenta-se como } \\
\text { um desafio e como } \\
\text { proposta desta } \\
\text { política. }\end{array}$ & $\begin{array}{l}\text { Acesso equitativo a } \\
\text { serviços de saúde - } \\
\text { apresenta-se como } \\
\text { um desafio e como } \\
\text { proposta desta } \\
\text { política. }\end{array}$ & $\begin{array}{l}\text { Apresenta-se de forma } \\
\text { transversal dentro da } \\
\text { política. }\end{array}$ & $\begin{array}{l}\text { Proposta da política } \\
\text { - atenção à saúde } \\
\text { de alta qualidade } \\
\text { para as pessoas } \\
\text { durante o processo de } \\
\text { envelhecimento. }\end{array}$ \\
\hline $\begin{array}{l}\text { Estatuto do } \\
\text { Idoso (BRASIL, } \\
2003)^{3}\end{array}$ & $\begin{array}{l}\text { Consta na descrição } \\
\text { da Lei } \\
\text { - Art. 15: É } \\
\text { assegurada a atenção } \\
\text { integral à saúde do } \\
\text { idoso... Garantindo- } \\
\text { lhe o acesso universal } \\
\text { e igualitário. }\end{array}$ & $\begin{array}{l}\text { Consta na } \\
\text { descrição da Lei } \\
\text { - Art. 15: Atenção } \\
\text { especializada para } \\
\text { idosos acamados, } \\
\text { com deficiência ou } \\
\text { limitação. }\end{array}$ & $\begin{array}{l}\text { Consta na descrição da Lei } \\
\text { - Art. 15: É assegurada a } \\
\text { atenção integral à saúde } \\
\text { do idoso, por intermédio } \\
\text { do Sistema Único de } \\
\text { Saúde - SUS }\end{array}$ & $\begin{array}{l}\text { Consta na descrição } \\
\text { da Lei } \\
\text { - Art. 15: Atenção } \\
\text { especializada para } \\
\text { idosos acamados, } \\
\text { com deficiência ou } \\
\text { limitação. }\end{array}$ \\
\hline $\begin{array}{l}\text { Toward } \\
\text { age-friendly } \\
\text { primary health } \\
\text { care (WHO, } \\
2004)^{4}\end{array}$ & $\begin{array}{l}\text { Refere como } \\
\text { princípio } \\
\text { fundamental } \\
\text { da APS- Acesso } \\
\text { universal e amigável. }\end{array}$ & $\begin{array}{l}\text { Refere como } \\
\text { princípio } \\
\text { fundamental } \\
\text { da APS- Acesso } \\
\text { equitativo. }\end{array}$ & $\begin{array}{l}\text { Propõem que barreiras } \\
\text { sejam quebradas visando a } \\
\text { integralidade do cuidado. }\end{array}$ & $\begin{array}{l}\text { Coloca a qualidade } \\
\text { do cuidado como um } \\
\text { critério essencial a ser } \\
\text { buscado pelos serviços } \\
\text { de saúde. }\end{array}$ \\
\hline $\begin{array}{l}\text { Pacto pela } \\
\text { Vida (BRASIL, } \\
2006)^{5}\end{array}$ & $\begin{array}{l}\text { Propõem a } \\
\text { reorganização } \\
\text { do processo de } \\
\text { acolhimento à } \\
\text { pessoa idosa, como } \\
\text { forma de qualificar o } \\
\text { acesso. }\end{array}$ & $\begin{array}{l}\text { Diretriz do Pacto: } \\
\text { Acolhimento } \\
\text { preferencial em } \\
\text { unidades de saúde. }\end{array}$ & $\begin{array}{l}\text { Diretrizes do Pacto: } \\
\text { i) Atenção integral e } \\
\text { integrada à saúde da } \\
\text { pessoa idosa; e ii) } \\
\text { Estímulo às ações } \\
\text { intersetoriais, visando à } \\
\text { integralidade da atenção. }\end{array}$ & $\begin{array}{l}\text { Diretriz do Pacto: } \\
\text { Provimento de } \\
\text { recursos capazes de } \\
\text { assegurar qualidade } \\
\text { da atenção à saúde da } \\
\text { pessoa idosa. }\end{array}$ \\
\hline $\begin{array}{l}\text { Política } \\
\text { Nacional se } \\
\text { Saúde da Pessoa } \\
\text { Idosa (BRASIL, } \\
2006)^{6}\end{array}$ & $\begin{array}{l}\text { Cita que o acesso } \\
\text { deve ser universal e } \\
\text { facilitado. }\end{array}$ & $\begin{array}{l}\text { Refere a diretriz } \\
\text { do SUS como } \\
\text { referencial da } \\
\text { política. }\end{array}$ & $\begin{array}{l}\text { Diretrizes da PNSPI: } \\
\text { i) Atenção integral e } \\
\text { integrada à saúde da } \\
\text { pessoa idosa; e ii) } \\
\text { Estímulo às ações } \\
\text { intersetoriais, visando à } \\
\text { integralidade da atenção. }\end{array}$ & $\begin{array}{l}\text { Diretriz preconizada } \\
\text { pela PNSPI: } \\
\text { Provimento de } \\
\text { recursos capazes de } \\
\text { assegurar qualidade } \\
\text { da atenção à saúde da } \\
\text { pessoa idosa. }\end{array}$ \\
\hline
\end{tabular}

$7,23)$ e escolaridade média de 6 anos $( \pm 3,81)$ de estudos. A renda familiar média foi de $\mathrm{R} \$ 2346,00$ $( \pm \mathrm{R} \$ 2450,00)$. As condições de saúde geral dos idosos demonstraram alta prevalência de morbidades, sendo estas ainda maiores quando apenas os usuários da APS foram avaliados. Quanto às condições de saúde bucal, foram encontrados poucos indivíduos com dentição funcional $(\geq$ 20 dentes) e a presença de restos radiculares teve prevalência alta (Tabela 1).

O Coeficiente $\alpha$ de Cronbach calculado para a avaliação de fidedignidade das escalas foram, sucessivamente: 0,75 - MEEM, 0,76 - GDS, e valores entre 0,73 e 0,96 foram calculados para as subescalas do PCATool Brasil.

\section{Etapa - \\ Resultados do Censo das Unidades de Saúde}

A Distribuição das características das Unidades de Saúde e das equipes de APS dos distritos sanitários Lomba e Partenon podem ser observadas na Tabela 2. 
Quadro 3. Análise documental das políticas e protocolos nacionais e internacionais que envolvem a saúde do idoso, publicadas entre 2007-2012.

\begin{tabular}{|c|c|c|c|c|}
\hline & Acesso universal & Equidade & Integralidade & Qualidade do cuidado \\
\hline \begin{tabular}{l|} 
Caderno \\
de Atenção \\
Básica No $19-$ \\
Envelhecimento \\
e Saúde da \\
Pessoa Idosa \\
$($ BRASIL, 2007)
\end{tabular} & $\begin{array}{l}\text { Preconiza que a } \\
\text { universalidade } \\
\text { deve ser um } \\
\text { princípio que } \\
\text { oriente o cuidado } \\
\text { na atenção } \\
\text { primária à saúde. }\end{array}$ & $\begin{array}{l}\text { Preconiza que a } \\
\text { equidade deve ser } \\
\text { um princípio que } \\
\text { oriente o cuidado } \\
\text { na atenção } \\
\text { primária à saúde. }\end{array}$ & $\begin{array}{l}\text { Um dos objetivos do } \\
\text { caderno é a busca pela } \\
\text { abordagem integral } \\
\text { no cuidado na atenção } \\
\text { primária à saúde. }\end{array}$ & $\begin{array}{l}\text { Propõem um protocolo } \\
\text { de gerenciamento do } \\
\text { cuidado a fim de zelar pela } \\
\text { qualidade do cuidado. } \\
\text { Propõem também outros } \\
\text { protocolos visando à } \\
\text { qualificação do cuidado. }\end{array}$ \\
\hline \begin{tabular}{l|} 
Age-friendly \\
Primary Health \\
Care Centres \\
Toolkit (WHO, \\
$2008)^{8}$
\end{tabular} & & & $\begin{array}{l}\text { Estabelece protocolos na } \\
\text { busca pela abordagem } \\
\text { integral do cuidado } \\
\text { em relação à saúde do } \\
\text { idoso. }\end{array}$ & $\begin{array}{l}\text { Define protocolos para a } \\
\text { qualificação do cuidado e } \\
\text { dos ambientes físicos dos } \\
\text { serviços de APS de acordo } \\
\text { com as demandas dos } \\
\text { idosos. }\end{array}$ \\
\hline \begin{tabular}{|l|} 
Atenção à \\
saúde da \\
pessoa idosa e \\
envelhecimento- \\
Série Pactos \\
pela Saúde 2006 \\
- v.12 (BRASIL, \\
$2010)^{9}$
\end{tabular} & & & & $\begin{array}{l}\text { Traz os referenciais } \\
\text { nacionais (Pacto e } \\
\text { Estatuto) e Internacionais } \\
\text { (Envelhecimento } \\
\text { Ativo) como apoio na } \\
\text { qualificação da atenção à } \\
\text { saúde do idoso. }\end{array}$ \\
\hline
\end{tabular}

\section{$4^{\text {a }}$ Etapa - Confrontação}

\section{Acesso universal}

Em relação ao uso dos serviços, 46,5\% dos idosos habitualmente acessam US de APS, e somente $18 \%$ costuma acessar tais serviços para atenção à saúde bucal. Quanto à efetividade do primeiro contato dos serviços acessados, foi possível observar que 53,9\% estavam de acordo com o preconizado. Quando apenas os serviços de APS foram avaliados, somente $42,4 \%$ dos serviços estavam orientados para a APS (Tabela 1).

A acessibilidade a serviços de saúde bucal foi avaliada através do censo, e apontou que embora a maioria das US tenha consultório odontológico, ainda existem algumas sem e outras que possuem consultório não acessíveis para cadeirantes (Tabela 2 ).

\section{Equidade}

A grande maioria das US avaliadas (89,5\%) possuía o agendamento telefônico como forma de acesso prioritário para os idosos (Tabela 2).

\section{Integralidade}

Apenas 25,6\% dos serviços acessados pelos idosos foram considerados efetivos quanto à integralidade, sendo que tal percentagem caiu ao tratar-se apenas de US de APS (23,9\%) (Tabela 1).

\section{Qualidade do Cuidado}

Analisando-se a orientação dos serviços acessados para os atributos essenciais da APS foi possível observar que apenas um quarto do total podia ser considerado efetivo $(25,8 \%)$. Sendo que quando apenas serviços de APS foram avaliados, este percentual caiu para $22,9 \%$. Ao incluir os atributos derivados na análise, a porcentagem de serviços com orientação para a APS caiu ainda mais (Tabela 1).

No que tange à qualidade das estruturas físicas, em relação ao escore geral, as US mostraram-se predominantemente inadequadas (57,9\%), da mesma forma que nos quesitos isolados de sinalização e acessibilidade de estruturas físicas (Tabela 2).

Ainda em relação às US e equipes de saúde, foi possível observar que: a maioria das equipes possuía pelo menos um profissional que já havia recebido algum tipo de capacitação relacionada à saúde do idoso (Tabela 2). 
Tabela 1. Descrição das características da amostra em relação a variáveis sóciodemográficas, agravos de saúde e percepções segundo idosos moradores dos distritos Partenon/Lomba de Porto Alegre-Brasil.

\begin{tabular}{|c|c|c|}
\hline & $\begin{array}{c}\mathrm{N}=862 \\
\mathrm{~m}(\mathrm{dp}), \mathrm{N}(\%)^{*}\end{array}$ & $\begin{array}{c}\mathrm{N}=401 \\
\mathrm{~m}(\mathrm{dp}), \mathrm{N}(\%)^{* *}\end{array}$ \\
\hline \multicolumn{3}{|l|}{ Características da amostra } \\
\hline Idade & $69,78(7,23)$ & $69,22(6,84)$ \\
\hline Escolaridade & $6,09(3,81)$ & $4,91(3,39)$ \\
\hline No de dependentes da renda familiar & $2,93(1,76)$ & $3,02(1,87)$ \\
\hline \multicolumn{3}{|l|}{ Renda } \\
\hline Até 2 salários mínimos & $308(35,7)$ & $203(50,6)$ \\
\hline Mais de 2 salários & $554(64,3)$ & $198(49,4)$ \\
\hline \multicolumn{3}{|l|}{ Renda suficiente } \\
\hline Sim & $379(44,0)$ & $123(30,7)$ \\
\hline Não & $483(56,0)$ & $278(69,3)$ \\
\hline \multicolumn{3}{|l|}{ Sexo } \\
\hline Masculino & $277(32,1)$ & $122(30,4)$ \\
\hline Feminino & $585(67,9)$ & $279(69,6)$ \\
\hline \multicolumn{3}{|l|}{ Tipo de serviço que acessa habitualmente } \\
\hline \multicolumn{3}{|l|}{ Serviço de Saúde geral } \\
\hline UBS & $312(36,2)$ & $312(77,8)$ \\
\hline USF & $89(10,3)$ & $89(22,2)$ \\
\hline Outros serviços & $461(53,4)$ & - \\
\hline \multicolumn{3}{|l|}{ Serviço de Saúde Bucal } \\
\hline UBS & $123(14,3)$ & $100(24,9)$ \\
\hline USF & $32(3,7)$ & $28(7)$ \\
\hline Outros serviços & $695(80,4)$ & $264(65,8)$ \\
\hline Nunca foi ao dentista & $12(1,4)$ & $9(2,2)$ \\
\hline \multicolumn{3}{|l|}{ Condições de Saúde } \\
\hline \multicolumn{3}{|l|}{ Estado mental (MEEM) } \\
\hline Escore $<$ média & $291(33,8)$ & $157(39,2)$ \\
\hline Escore $\geq$ média & $571(66,2)$ & $244(60,8)$ \\
\hline \multicolumn{3}{|l|}{ Sintomatologia depressiva } \\
\hline Com sintomatologia & $246(28,5)$ & $142(35,4)$ \\
\hline Sem sintomatologia & $616(71,5)$ & $259(64,6)$ \\
\hline \multicolumn{3}{|l|}{ Hipertensão } \\
\hline Sim & $501(58,1)$ & $241(60,1)$ \\
\hline Não & $361(41,9)$ & $160(39,9)$ \\
\hline \multicolumn{3}{|l|}{ Diabetes } \\
\hline Sim & $180(20,9)$ & $89(22,2)$ \\
\hline Não & $682(79,1)$ & $312(77,8)$ \\
\hline \multicolumn{3}{|l|}{ Dor nas costas } \\
\hline Sim & $336(39,0)$ & $179(44,6)$ \\
\hline Não & $526(61,0)$ & $222(55,4)$ \\
\hline \multicolumn{3}{|l|}{ Número de dentes ${ }^{\dagger}$} \\
\hline 0 & $251(31)$ & $138(35,7)$ \\
\hline $1-19$ & $435(53,7)$ & $207(53,5)$ \\
\hline$\geq 20$ & $124(15,3)$ & $42(10,9)$ \\
\hline \multicolumn{3}{|l|}{ Restos Radiculares $^{\dagger}$} \\
\hline 0 & $696(85,9)$ & $331(85,5)$ \\
\hline$\geq 1$ & $114(14,1)$ & $56(14,5)$ \\
\hline
\end{tabular}

\section{Avaliação transversal às categorias}

A maioria das equipes das US fazia visitas domiciliares a acamados e possuía algum tipo de grupo para idosos (Tabela 2).

\section{Discussão}

Este estudo possui um caráter inédito sob diversos aspectos: propõe-se a uma confrontação entre 


\begin{tabular}{lcc}
\hline Tabela 1. continuação & & \\
\hline & $\mathbf{N}=\mathbf{8 6 2}$ & $\mathbf{N}=\mathbf{4 0 1}$ \\
$\mathbf{m}(\mathbf{d p}), \mathbf{N}(\%)^{*}$ & $\mathbf{m}(\mathbf{d p}), \mathbf{N}(\%)^{* *}$ \\
\hline Orientação para a APS & & \\
$\quad$ Primeiro Contato & $465(53,9)$ & $170(42,4)$ \\
$\quad$ Com orientação & $397(46,1)$ & $231(57,6)$ \\
$\quad$ Sem orientação & & $184(45,9)$ \\
Longitudinalidade & $464(63,8)$ & $217(54,1)$ \\
$\quad$ Com orientação & $398(46,2)$ & $96(23,9)$ \\
$\quad$ Sem orientação & $220(25,6)$ & $305(76,1)$ \\
Integralidade & $641(74,4)$ & $179(44,6)$ \\
$\quad$ Com orientação & & $219(54,6)$ \\
$\quad$ Sem orientação & $372(43,5)$ & \\
Coordenação & $484(56,5)$ & $92(22,9)$ \\
$\quad$ Com orientação & & $309(77,1)$ \\
$\quad$ Sem orientação & $222(25,8)$ & $81(20,2)$ \\
Orientação para a APS - Quanto aos atributos essenciais. & $640(74,2)$ & $320(79,8)$ \\
$\quad$ Com orientação & & \\
$\quad$ Sem orientação & $155(18,1)$ & $706(81,9)$ \\
Orientação para a APS - Quanto aos atributos gerais. & & \\
$\quad$ Com orientação & & \\
$\quad$ Sem orientação & & \\
\hline Dados do total da amostra. * Dados dos participantes que utilizam serviços de APS. ${ }^{\dagger}$ Os participantes que se recusaram a fazer o \\
exame de saúde bucal foram excluídos desta análise.
\end{tabular}

teoria e prática relacionadas com a Saúde do Idoso, avaliação de processos e ambientes físicos, e abordagem da saúde bucal sob a perspectiva da integralidade do cuidado.

Os resultados mostraram um distanciamento entre as informações obtidas através dos documentos, e os dados empíricos apresentados. O Estatuto do Idoso garante a todo o idoso brasileiro acesso universal e igualitário à atenção integral à saúde, por intermédio do SUS. E, outros documentos analisados para o desenvolvimento da matriz teórica também evidenciaram a universalidade de acesso como parte integrante de suas bases conceituais ${ }^{2,3,5-7}$. No entanto, o que os dados empíricos mostram é uma realidade distinta: apenas metade dos idosos avaliados costuma acessar a APS do SUS, e tais números caem vertiginosamente quando se trata de saúde bucal.

Quanto à efetividade do primeiro contato dos idosos com os serviços, foi possível observar que apenas cerca de metade dos serviços acessados corresponde ao preconizado. Resultados da Pesquisa Nacional por Amostra de Domicílios (PNAD) mostram que houve melhoras significativas no acesso a serviços de APS nas últimas décadas para os idosos ${ }^{26}$, no entanto, apesar dos dados apontarem melhoras, ainda há muito a ser feito em relação à ampliação do acesso ${ }^{13,14,27}$.
Os resultados encontrados podem ser decorrentes de uma escolha por parte dos idosos, entretanto, há espaço para a reflexão a respeito da cobertura da SF, bem como da APS como um todo, em Porto Alegre. Costa e Ciosak ${ }^{28}$ colocam que para o idoso, principalmente o mais carente, qualquer dificuldade no acesso pode se tornar uma barreira capaz de bloquear ou interromper a continuidade do cuidado.

Já em relação à integralidade, evidências ${ }^{14,26,28-30}$ e a descrição das características da amostra demonstram a necessidade real deste atributo da APS no cotidianos da prática de cuidado, uma vez que os idosos possuem alta prevalência de morbidades e más condições de saúde bucal.

Ao avaliar-se a efetividade da integralidade do cuidado, foi possível observar problemas significativos. Algumas explicações podem estar relacionadas: evidências mostram que UBS funcionam ainda sob a lógica biomédica focada em enfermidades e não em pessoas e possuem escores mais baixos de integralidade quando comparados às USF e às unidades de ensino em serviço que dispõem de residência multiprofissional ${ }^{31}$. Tal achado possivelmente esteja relacionado com o desafio que constitui o rompimento do modus operandi de tais estabelecimentos. Realidade esta que também pode ser observada no setor privado que possui a 
Tabela 2. Distribuição das características das Unidades de Saúde e das equipes de APS dos distritos sanitários Lomba e Partenon.

\begin{tabular}{|c|c|}
\hline & $\mathrm{N}(\%)$ \\
\hline \multicolumn{2}{|l|}{ Características das Unidades } \\
\hline \multicolumn{2}{|l|}{ Andares } \\
\hline 1 & $16(84,2)$ \\
\hline 2 & $2(10,5)$ \\
\hline 3 & $1(5,3)$ \\
\hline \multicolumn{2}{|l|}{ Modelo de APS } \\
\hline UBS & $8(42,1)$ \\
\hline USF & $11(57,9)$ \\
\hline \multicolumn{2}{|l|}{ Estruturas Físicas } \\
\hline \multicolumn{2}{|l|}{ Estruturas Geral } \\
\hline Inadequado & $11(57,9)$ \\
\hline Parcialmente Adequado & $8(42,1)$ \\
\hline \multicolumn{2}{|l|}{ Sinalização } \\
\hline Sinalização Geral & $11(57,9)$ \\
\hline Inadequado & $8(42,1)$ \\
\hline \multicolumn{2}{|l|}{ Parcialmente Adequado } \\
\hline \multicolumn{2}{|l|}{ Checagem Geral } \\
\hline \multicolumn{2}{|l|}{ Escore geral } \\
\hline Inadequado & $10(52,6)$ \\
\hline Parcialmente Adequado & $9(47,4)$ \\
\hline \multicolumn{2}{|l|}{ Questões adicionais } \\
\hline \multicolumn{2}{|l|}{ Capacitação em Atenção à Saúde do Idoso } \\
\hline 0 & $5(26,3)$ \\
\hline 1 & $3(15,8)$ \\
\hline 2 ou mais profissionais capacitados & $11(57,9)$ \\
\hline \multicolumn{2}{|l|}{ Agendamento por telefone } \\
\hline Realiza & $17(89,5)$ \\
\hline Não realiza & $2(10,5)$ \\
\hline \multicolumn{2}{|l|}{ Grupo para Idosos } \\
\hline Realiza & $14(73,7)$ \\
\hline Não realiza & $5(26,3)$ \\
\hline \multicolumn{2}{|l|}{ Visitas domiciliares } \\
\hline Realiza & $18(94,7)$ \\
\hline Não realiza & $1(5,3)$ \\
\hline \multicolumn{2}{|l|}{ Possui consultório odontológico } \\
\hline Sim & $13(68,4)$ \\
\hline Não & $6(31,6)$ \\
\hline \multicolumn{2}{|l|}{ O consultório é acessível para cadeirante } \\
\hline $\operatorname{Sim}$ & $10(76,9)$ \\
\hline Não & $3(23,1)$ \\
\hline
\end{tabular}

concepção mercadológica da saúde. Um estudo de avaliação do setor de saúde suplementar observou que empresas operadoras de planos privados de saúde não demonstram interesse em proporcionar aos seus segurados um cuidado integral e o monitoramento de todas as etapas do envelhecimento ${ }^{32}$.

A mudança em busca da integralidade exige o rompimento do modelo biomédico através de políticas de priorização da SF, de mudança na formação de recursos humanos ${ }^{33}$ e na lógica de atenção que desconsidera o uso de protocolos e evidências na prática do cuidado na APS. Além disso, a própria SF em Porto Alegre também possui limitações que possivelmente dificultem o processo de trabalho e a busca pelo cuidado integral, tais como a precarização de contratos de trabalho ${ }^{34}$ e a ausência de Núcleos de Apoio à Saúde da Família (NASF) ${ }^{35}$.

A presença de restos radiculares em 14\% da amostra estudada também é uma evidência de que o cuidado oferecido aos idosos na APS do SUS não é provido de forma integral. Tal resultado indica que um número expressivo de idosos não tem recebido cuidados odontológicos. Restos radiculares são consequências de um avançado processo de cárie que destrói as coroas dentárias deixando apenas raízes. Cáries extensas podem causar dor e desconforto, e são focos sépticos que podem implicar em infecções ${ }^{36}$.

Quanto à equidade, o censo observou que os idosos possuíam o dispositivo de agendamento por telefone como forma de acesso prioritário, direito assegurado por meio da Lei Municipal 10.819 de $2010^{37}$. O acesso facilitado é importante uma vez que a dificuldade de deambulação, audição ou visão, e, até mesmo, incontinência urinária podem inviabilizar a espera. No entanto, observou-se que nos DS estudados a lei não estava sendo cumprida em sua totalidade.

Voltando o olhar para a APS, é possível observar através da matriz teórico-lógica desenvolvida que tal nível de atenção é visto como lócus privilegiado de atenção à saúde do idoso ${ }^{4,7,8}$. A APS possui a particularidade de conseguir prevenir condições incapacitantes e prevalentes na terceira idade caso consiga estar corretamente orientada pelos seus atributos. A garantia dos atributos da APS implica em melhores indicadores de saúde ${ }^{24}$ e possivelmente também em condições propícias para um envelhecimento ativo, uma vez que o indivíduo terá sua saúde integralmente assistida, de forma que condições incapacitantes venham a ser prevenidas e outras manejadas.

Evidências sobre a avaliação da qualidade da APS acessada por idosos não foram encontradas, no entanto, os resultados obtidos mostram que apenas uma pequena parte dos serviços de saúde acessados foi capaz de cumprir o preconizado para a APS. Tal resultado demonstra que apesar das Políticas e Protocolos ${ }^{4,7.8}$, algumas lacunas ainda inviabilizam a efetividade da APS. Entre elas é possível apontar: restrição no acesso, desarticulação da rede de cuidados, limitação de escopo de serviços oferecidos e despreparo das equipes para necessidades específicas que acometem os idosos. 
Além disso, os resultados do censo das US também apontam para inadequação de acessibilidade e sinalização das US e da identificação das equipes, realidade já observada em outros estudos ${ }^{38,39}$. A presença de degraus, a falta de corrimãos, rampas, banheiros adaptados para os cadeirantes, salas de espera inadequadas, instalações sem identificação para portadores de limitações visuais ou auditivas constituem barreiras no acesso aos serviços de saúde e na continuidade do cuidado.

O fato da amostra ser restrita a dois DS de Porto Alegre sugere que novas avaliações com amostras representativas de cidades poderiam trazer resultados mais amplos e capazes de estabelecer um paralelo em relação ao que está proposto nas políticas.

Após o desenvolvimento deste estudo, vale destacar que os documentos são coerentes com os marcos teóricos do SUS e apresentam-se bem estruturados. No entanto, na prática, o acesso à APS do SUS ainda não pode ser considerado universal aos idosos e poucos serviços podem ser considerados de qualidade no que tange à orientação para a APS. Pelo que se observa, realmente parece existir um gargalo no qual idosos com uma ampla carga de morbidades desafiam o sistema para dar conta das suas necessidades, sem encontrar o devido respaldo.

Neste ínterim, algumas mudanças estruturais devem ser realizadas para que a saúde do idoso seja tratada com a dignidade que merece. Como já foi dito, o acesso à APS e, principalmente, à saúde da família, deve ser ampliado. No entanto, não será a única solução. É preciso preparar idosos, famílias e comunidades para o enfrentamento da realidade que está posta e para isto será preciso contar com serviços de saúde de qualidade na atenção básica, na média e alta complexidade e na saúde suplementar. Para tanto, há necessidade de que todo o setor saúde esteja apto, tanto na esfera pública quanto na privada, para promover saúde, visando ao envelhecimento ativo. Tal perspectiva implica em colocar a Saúde do Idoso na agenda das políticas públicas em diversos âmbitos: Atenção Básica, Programa de Melhoria do Acesso e da Qualidade do SUS, Saúde Suplementar, Pesquisa em Saúde, e, Formação de Recursos Humanos.

Urge que algumas medidas passem a ser postas em prática imediatamente, tais como: garantia de equidade, de acessibilidade e de qualificação do se- tor saúde no que tange às necessidades dos idosos. O uso rotineiro de protocolos de atenção à saúde do idoso, já consagrados através de evidências, seria uma das ferramentas para a qualificação. Além disso, a inclusão da saúde bucal na pauta do cuidado integral e no cotidiano de práticas também parece ser imperativa neste momento. Por último, mas não menos importante, a garantia de um controle social efetivo que dê voz aos próprios idosos deve ser vista como uma prioridade, uma vez que a qualificação do sistema deve ser feita para e com os seus usuários e não apenas a partir de indicadores de desempenho.

\section{Colaboradores}

AB Martins trabalhou na concepção, na elaboração da pesquisa e na redação de todo o texto; $O P$ D'Avila na coleta de dados da pesquisa e no desenvolvimento da metodologia da pesquisa e do manuscrito; JB Hilgert na concepção do estudo e na redação final; e, FN Hugo na orientação de todo o desenvolvimento do estudo e ma redação do manuscrito.

\section{Agradecimentos}

Este artigo faz parte de um projeto financiado pela Fundação de Amparo à Pesquisa do Estado do Rio Grande do Sul (FAPERGS) e pela Coordenação de Aperfeiçoamento de Pessoal de Nível Superior (Capes) - bolsa de doutorado de Aline Blaya Martins vinculada ao PPG-ODO UFRGS. 


\section{Referências}

1. Brasil. Portaria $\mathrm{n}^{\circ} 1395 / \mathrm{GM}$, de 10 de dezembro de 1999. Política de Saúde do Idoso. Diário Oficial da União 1999; 13 dez.

2. World Health Organization (WHO). Active Ageing: A Policy Framework. Geneva: WHO; 2002.

3. Brasil. Presidência da República. Lei 10.741 , de $1^{\circ}$ de outubro de 2003. Dispõe sobre o Estatuto do Idoso e dá outras providências. Diário Oficial da União 2003; 3 out.

4. World Health Organization (WHO). Towards Age-friendly Primary Health Care. Geneva: WHO; 2004.

5. Brasil. Ministério da Saúde (MS). Portaria n 399/GM, de 22 de fevereiro de 2006. Divulga o Pacto pela Saúde 2006 - Consolidação do SUS e aprova as Diretrizes Operacionais do Referido Pacto. Diário Oficial da União 2006; 23 fev.

6. Brasil. Portaria no 2.528 , de 19 de outubro de 2006 . Aprova a Política Nacional de Saúde da Pessoa Idosa. Diário Oficial da União 2006; 20 out.

7. Brasil. Ministério da Saúde (MS). Caderno de Atenção Básica no 19: Envelhecimento e Saúde da Pessoa Idosa. Brasília: MS; 2007.

8. World Health Organization (WHO). Age-friendly Primary Health Care Centres Toolkit. Geneva: WHO; 2008.

9. Brasil. Ministério da Saúde (MS). Secretaria de Atenção à Saúde. Departamento de Ações Programáticas e Estratégicas. Área Técnica Saúde do Idoso. Atenção à saúde da pessoa idosa e envelhecimento. Brasília: MS; 2010.

10. Instituto Brasileiro de Geografia e Estatística (IBGE). Sinopse do Censo Demográfico 2010. Rio de Janeiro: IBGE; 2011.

11. Brasil. Constituição da República Federativa do Brasil de 1988. Diário Oficial da União 1988; 5 out.

12. Mendonça CS. Saúde da Família, agora mais do que nunca! Cien Saude Colet 2009; 14(Supl. 1):1493-1497.

13. Louvison MCP, Lebrão ML, Duarte YAO, Santos JLF, Malik AM, Almeida ES. Desigualdades no uso e acesso aos serviços de saúde entre idosos do município de São Paulo. Inequalities in access to health care services and utilization for the elderly in São Paulo, Brazil. Rev Saude Publica 2008; 42(4):733-740.

14. Marin MJS, Cecílio LCO. Necessidades de saúde de idosos de uma Unidade de Saúde da Família. Rev Bras Geriatr Gerontol 2009; 12(1):63-76.

15. Brasil. Lei no 8.080, de 19 de setembro de 1990. Dispõe sobre as condições para a promoção, proteção e recuperação da saúde, a organização e o funcionamento dos serviços correspondentes e dá outras providências. Diário Oficial da União 1990; 20 set.

16. Folstein MF, Folstein SE, McHugh PR. "Mini-mental state". A practical method for grading the cognitive state of patients for the clinician. J Psychiatr Res 1975; 12(3):189-198.

17. Bertolucci PHF, Brucki SMD, Campacci SR, Juliano Y O mini-exame do estado mental em uma população geral: impacto da escolaridade. Arq Neuropsiquiatr 1994; 52(1):1-7.
18. Brasil. Ministério da Saúde (MS). Secretaria de Atenção em Saúde. Departamento de Atenção Básica. Manual do instrumento de avaliação da atenção primária à saúde: primary care assessment tool pcatool - Brasil. Brasília: MS; 2010.

19. Barros FC, Victora CG. Epidemiologia da Saúde Infantil: um manual para diagnósticos comunitários. $3^{\mathrm{a}}$ ed. São Paulo, Brasília: Hucitec, Unicef; 1998.

20. Yesavage JA, Brink TL, Rose TL, Lum O, Huang V, Adey $\mathrm{M}$, Leirer VO. Development and validation of a geriatric depression screening scale: a preliminary report. $J$ Psychiatr Res 1982; 17(1):37-49.

21. Almeida OP, Almeida SA. Short versions of the geriatric depression scale: a study of their validity for the diagnosis of a major depressive episode according to ICD-10 and DSM-IV. Int J Geriatr Psychiatry 1999; 14(10):858-865.

22. Organização Mundial de Saúde (OMS). Levantamentos em Saúde Bucal. São Paulo: Livraria e Editora Santos; 1999.

23. Agerberg G, Carlsson GE. Chewing ability in relation to dental and general health. Analyses of data obtained from a questionnaire. Acta Odontol Scand 1981; 39(3):147-153.

24. Starfield B. Atenção Primária: Equilíbrio entre necessidades de saúde, serviços e tecnologia. Brasília: Unesco, MS; 2002.

25. Shi L, Starfield B, Jihaong X. Validating of the adult primary care assessment tool. J Family Practice 2001; 50:161-175.

26. Lima Costa MF, Matos DL, Camargos VP, Macinko J. Tendências em dez anos das condições de saúde de idosos brasileiros: evidências da Pesquisa Nacional por Amostra de Domicílios (1998, 2003, 2008). Cien Saude Colet 2011; 16(9):3689-3696.

27. Kalache A. Fórum. Envelhecimento populacional e as informações de saúde do PNAD: demandas e desafios contemporâneos. Posfácio. Cad Saude Publica 2007; 23(10):2503-2505.

28. Costa MFBNA, Ciosak SI. Atenção integral na saúde do idoso no Programa Saúde da Família: visão dos profissionais de saúde. Rev Esc Enferm USP 2010; 44(2):437-444.

29. Hugo FN, Hilgert JB, de Sousa Mda L, da Silva DD, Pucca GA Jr. Correlates of partial tooth loss and edentulism in the Brazilian elderly. Community Dent Oral Epidemiol 2007; 35(3):224-232.

30. De Marchi RJ, Hilgert JB, Hugo FN, Santos CM, Martins AB, Padilha DM. Four-year incidence and predictors of tooth loss among older adults in a southern Brazilian city. Community Dent Oral Epidemiol 2012; 40(5):396405.

31. Castro RCL, Knauth DR, Harzheim E, Hauser L, Duncan BB. Avaliação da qualidade da atenção primária pelos profissionais de saúde: comparação entre diferentes tipos de serviços. Cad Saude Publica 2012; 28(9):1772-1784. 
32. Veras RP, Caldas CP, Araújo DV, Mendes RKW. A assistência suplementar de saúde e seus projetos de cuidado para com o idoso. Cien Saude Colet 2008; 13(4):1119-1126.

33. Conill EM. Ensaio histórico-conceitual sobre a Atenção Primária à Saúde: desafios para a organização de serviços básicos e da Estratégia Saúde da Família em centros urbanos no Brasil. Cad Saude Publica 2008; 24(Supl. 1):S7-27.

34. Leão MSV. Programa Saúde da Família: Dificuldade, méritos e contradições no Município de Porto Alegre [dissertação]. Porto Alegre: Universidade Federal do Rio Grande do Sul; 2009.

35. Brasil. Ministério da Saúde (MS). Secretaria de Atenção à Saúde. Departamento de Atenção Básica. Diretrizes do NASF: Núcleo de Apoio a Saúde da Família. Brasília: MS; 2010.

36. Shay K. Infectious Complications of Dental and Periodontal Diseases in the Elderly Population. Clin Infect Dis 2002; 34(9):1215-1223.

37. Porto Alegre. Prefeitura Municipal. Lei Municipal no 10.819 , de 13 de janeiro de 2010. Estabelece a possibilidade do agendamento telefônico de consultas para pacientes idosos e para pessoas com deficiências já cadastrados nas unidades de saúde do município de Porto Alegre e dá outras providências. Diário Oficial de Porto Alegre 2010; 14 jan.

38. Vasconcelos LR, Pagliuca LMF. Mapeamento da acessibilidade do portador de limitação física a serviços básicos de saúde. Esc Anna Nery R Enferm 2006; 10(3):494-500.

39. Siqueira FCV, Facchini LA, Silveira DS, Piccini RX, Thumé E, Tomasi E. Barreiras arquitetônicas a idosos e portadores de deficiência física: um estudo epidemiológico da estrutura física das unidades básicas de saúde em sete estados do Brasil. Cien Saude Colet 2009; 14(1):39-44.

Artigo apresentado em 02/06/2013

Aprovado em 27/07/2013

Versão final apresentada em 02/08/2013 